\title{
Answer to Quiz Electrocardiogram on page .. and case discussion
}

Correct answer is B:

B) Sinus rhythm with first-degree AV block (Fig. 2)

On last two complexes PQRST heart rate slows down and $P$ wave become visible. It appears from the $T$ wave "shadow» and PQ interval can be measured. It equals 360 milliseconds. Measurement is performed in last PQRST complex in lead II.

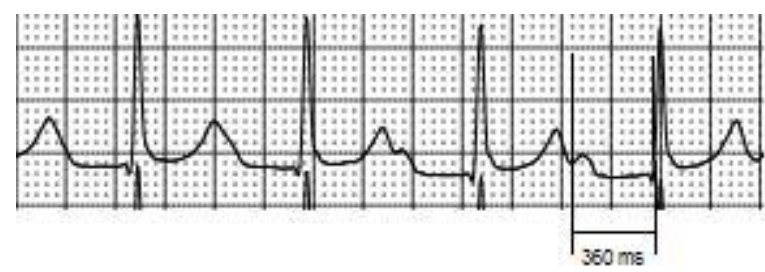

Figure 2. Electrocardiogram lead II fragment

\section{Discussion}

AV conduction disturbances are common complication of myocarditis that can occur up to $33 \%$ of cases. Higher-degree AV block is correlated with greater myocardial injury and reversibility of ECG changes reflect the severity of pathological changes. In most cases of myocarditis complicated by AV block there is no need of permanent pacemaker placement. However, low-degree AV block can sustain after acute stage of myocarditis for a long time $(1,2)$.

When differentiating between atrial flutter with 2:1 conduction, sinus tachycardia and supraventricular tachycardia, vagal maneuvers can be performed. Carotid sinus massage usually decreases AV conduction to $3: 1$ or more and the atrial activity can become visible on 12-lead ECG. In case of the paroxysm of supraventricular tachycardia, vagal maneuvers can be effective for the cessation of this arrhythmia. Carotid sinus massage combined with Valsalva maneuver are reported to be effective for sinus rhythm conversion in $27.7 \%$ patients with supraventricular tachycardias (3). Sinus tachycardia also can be slowed by vagal maneuvers. In most cases, this decrease in heart rate will be sufficient for better P-wave visualization.

Mykhaylo Sorokivskyy Danilo Hlacky University, Lviv, Ukraine

Peer-review: Internal

Conflict of interest: None declared

Authorship: M.S. Acknowledgement and funding: None declared.

\section{References:}

1. Nakashima H, Honda $\mathrm{Y}$, Katayama $T$. Serial electrocardiographic findings in acute myocarditis. Intern Med 1994; 33: 659-66.

2. Caughey RW, Humphrey JM, Thomas PE. High-Degree Atrioventricular Block in Child with Acute Myocarditis. Ochsner J 2014; 14: 244-7.

3. Lim SH, Anantharaman V, Teo WS, Goh, PP, Tan AT. Comparison of treatment of supraventricular tachycardia by Valsalva maneuver and carotid sinus massage. Ann Emerg Med 1988; 31: 30-5.

Address for Correspondence: Mykhaylo Sorokivskyy, Danilo Hlacky University, Lviv, Ukraine Email: msorokivskyy1@gmail.com Received: 07.04.2018 Accepted: 08.04.2018 doi: 10.24969/hvt.2018.59 